\title{
Cloning and expression of a novel catechol- 0 -methyltransferase in common marmosets
}

\author{
Shotaro UEHARA ${ }^{1)}$, Yasuhiro UNO ${ }^{2)^{*}}$, Takashi INOUE ${ }^{3)}$, Erika SASAKI ${ }^{3,4)}$ and \\ Hiroshi YAMAZAKI ${ }^{1)^{*}}$ \\ 1)Laboratory of Drug Metabolism and Pharmacokinetics, Showa Pharmaceutical University, Machida, Tokyo
194-8543, Japan \\ 2)Pharmacokinetics and Bioanalysis Center, Shin Nippon Biomedical Laboratories, Ltd., Kainan, Wakayama, \\ Japan \\ 3) Department of Applied Developmental Biology, Central Institute for Experimental Animals, Kawasaki, Japan \\ ${ }^{4)}$ Keio Advanced Research Center, Keio University, Minato-ku, Tokyo, Japan
}

J. Vet. Med. Sci.

79(2): 267-272, 2017

doi: 10.1292/jvms.16-0459

Received: 5 September 2016 Accepted: 14 November 2016 Published online in J-STAGE: 26 November 2016

\begin{abstract}
Catechol-O-methyltransferase (COMT) catalyzes the $O$-methylation of endogenous catechol amines and estrogens and exogenous catechol-type of drugs. A Parkinson's disease model of common marmoset (Callithrix jacchus) has been widely used in preclinical studies to evaluate inhibitory potential of new drug candidates on marmoset COMT. Despite COMT inhibitors could potentiate the pharmacological action of levodopa on Parkinson's disease in animal models, marmoset COMT CDNA has not yet been identified and characterized. In this study, a cDNA highly homologous to human COMT was cloned from marmoset livers. This CDNA encoded 268 amino acids containing a transmembrane region and critical amino acid residues for catalytic function. The amino acid sequences of marmoset COMT shared high sequence identity (90\%) with human COMT. COMT mRNA was expressed in all five tissues tested, including brain, lung, liver, kidney and small intestine, and was more abundant in marmoset liver and kidney. Membrane-bound COMT was immunochemically detected in livers and kidneys, whereas soluble COMT was detected in livers, similar to humans. These results indicated that the molecular characteristics of marmoset COMT were generally similar to the human ortholog.

KEY WORDS: marmoset, membrane-bound COMT, soluble COMT
\end{abstract}

Catechol- $O$-methyltransferase (COMT) is a magnesium-dependent methyltransferase catalyzing the $O$-methylation reactions using $S$-adenosyl-L-methionine as the methyl group donor [7]. COMT plays a role in the metabolism of not only key endogenous compounds, such as catechol-containing substrates, the catecholamines and steroid/thyroid hormones (e.g., dopamine, norepinephrine, epinephrine or catechol estrogen), but also many catechol drugs, such as levodopa, carbidopa, benserazide and apomorphine [5]. In humans, two isoforms of COMT exist; soluble COMT located in cytosolic fractions, and membrane-bound COMT located in rough endoplasmic reticulum by an anchoring $N$-terminal transmembrane region [6]. Soluble COMT is the predominant form in most tissues (most abundant in the liver and kidney), except for brain where membrane-bound COMT predominates. Membrane-bound COMT is reportedly involved in the inactivation of the catecholamine neurotransmitters, whereas soluble COMT plays an important role in the metabolic inactivation of endogenous and exogenous catechol compounds [3, 14].

Inhibition of COMT could enhance pharmacological action of levodopa in the treatment of Parkinson's disease, because COMT inactivates the catechol neurotransmitter dopamine and the drug levodopa by biotransformation. Inhibitory potential of new drug candidates on COMT has been evaluated in common marmoset (Callithrix jacchus) models treated with 1-methyl-4-phenyl1,2,3,6-tetrahydropyridine (MPTP), which are considered as a suitable non-human primate model for Parkinson's disease research $[4,11,16]$. Indeed, the co-administration of entacapone with levodopa reportedly produced the continuous dopaminergic stimulation and reduced the levodopa-induced dyskinesia induction in the animal models [13, 18]. However, DNA microarray analysis has indicated that COMT transcript was absent in prefrontal cortices of marmosets and cynomolgus monkeys, unlike humans [8]. On the other hand, Zeng et al. [17] detected COMT mRNA and protein in marmoset liver and brain. In spite of the importance of marmoset models in Parkinson's disease research, full-length cDNA sequences of marmoset COMT have not been identified and characterized.

To clarify the pharmacokinetics of drugs in marmosets at the molecular level, we have identified and characterized cytochrome P450 (P450) enzymes in marmosets [15]. The catalytic function and expression of P450s were relatively conserved between

*Correspondence to: Uno, Y., Pharmacokinetics and Bioanalysis Center, Shin Nippon Biomedical Laboratories, Ltd., 16-1 Minami Akasaka, Kainan 642-0017, Japan. e-mail: uno-yasuhiro@snbl.co.jp; Yamazaki, H., Showa Pharmaceutical University, 3-3165 Higashi-tamagawa Gakuen, Machida, Tokyo 194-8543, Japan.e-mail: hyamazak@ac.shoyaku.ac.jp

(O2017 The Japanese Society of Veterinary Science

This is an open-access article distributed under the terms of the Creative Commons Attribution Non-Commercial No Derivatives (by-ncnd) License <http://creativecommons.org/licenses/by-nc-nd/4.0/>. 
marmosets and humans. However, non-P450 drug metabolizing enzymes remained to be analyzed. In this study, we successfully isolated marmoset COMT cDNA from livers, based on the gene sequence from the marmoset genome data. The gene structure, sequence identity, phylogenetic relationship and tissue expression pattern of COMT in marmosets are reported herein.

\section{MATERIALS AND METHODS}

\section{Chemicals and antibodies}

Custom oligonucleotides were synthesized by Sigma Genosys Japan (Ishikari, Japan). Polyclonal anti-human COMT antibodies (FL-271), monoclonal anti-avian $\beta$-actin antibodies (C4) and goat anti-rabbit and goat anti-mouse IgG horseradish peroxidase conjugated secondary antibodies were purchased from Santa Cruz Biotechnology (Santa Cruz, CA, U.S.A.). All other solvents and reagents used were highest grades commercially available.

\section{Preparation of homogenates from marmoset tissues}

Marmoset tissue homogenates were prepared from brain, lung, liver, kidney and small intestine (jejunum and ileum) of 6 marmosets ( 3 males and 3 females, 2-6 years of age) housed with balanced food for marmosets (CMS-1; CLEA Japan, Kawasaki, Japan) at the Central Institution for Experimental Animals (Kawasaki, Japan). This study was approved by its Institutional Animal Care and Use Committee. Briefly, marmoset brains and livers were homogenized over wet ice in extraction buffer $(0.1 \mathrm{M}$ Tris-HCl, pH 7.4, containing 0.1 M KCl, $1.0 \mathrm{mM}$ EDTA, 0.005\% aprotinin and $0.001 \%$ leupeptin). Protein concentrations were determined using the BCA Protein Assay Kit (Thermo Fisher Scientific, Yokohama, Japan), and crude homogenates were stored in $20 \%$ glycerol at $-80^{\circ} \mathrm{C}$.

\section{COMT cDNA cloning}

Total RNA was extracted from marmoset livers using an RNeasy Mini Kit (Qiagen, Valencia, CA, U.S.A.). For first-strand cDNA preparation, reverse transcription (RT) was performed in a mixture containing $1 \mu \mathrm{g}$ of total RNA from marmoset livers, SuperScript III RT reverse transcriptase (Invitrogen, Carlsbad, CA, U.S.A.), anchored oligo (dT) primer (Invitrogen) and RNase OUT (Invitrogen) at $50^{\circ} \mathrm{C}$ for $60 \mathrm{~min}$. For cDNA cloning, polymerase chain reaction (PCR) was performed under the action of KOD-Plus-Neo DNA polymerase (Toyobo, Osaka, Japan) with an ABI GeneAmp PCR System 2720 thermocycler (Applied Biosystems, Foster City, CA, U.S.A.). Amplification conditions were 2 min at $94^{\circ} \mathrm{C}$ and 30 cycles of $98^{\circ} \mathrm{C}$ for 15 $\mathrm{sec}, 60^{\circ} \mathrm{C}$ for $30 \mathrm{sec}$ and $68^{\circ} \mathrm{C}$ for $2 \mathrm{~min}$, followed by a 7 -min extension at $68^{\circ} \mathrm{C}$. The PCR primers used were cjCOMT (5rt1) 5'-AGAAATTACATCTGCTTTGCTGC-3' and cjCOMT (3rt1) 5'-GCATTTAGGACACAGCCCAG-3'. Purified PCR products were cloned into pGEM-T easy vectors using a pGEM-T Easy Vector System (Promega, Madison, WI, U.S.A.). The sequence verification of insert was carried out using an ABI PRISM BigDye Terminator v3.0 Ready Reaction Cycle Sequencing Kit (Applied Biosystems) with an ABI PRISM 3730 DNA Analyzer (Applied Biosystems).

\section{Sequence analyses}

Gene structure was analyzed by BLAT (UCSC Genome Bioinformatics, University of California, Santa Cruz, CA, U.S.A.). The homology of amino acid sequences was analyzed using BLAST (National Center for Biotechnology Information, Bethesda, MD, U.S.A.). Multiple alignment of amino acid sequences was performed using a Genetyx system (Software Development, Tokyo, Japan). The phylogenetic analysis was conducted using the neighbor-joining method with DNASIS Pro (Hitachi Software, Tokyo, Japan). The COMT amino acid sequences for sequence analysis were found in GenBank: human COMT (NP_000745), rhesus monkey COMT (NP_001247941), pig COMT (NP_001182259), rat COMT (NP_036663), mouse COMT (NP_001104532) and zebrafish COMT (NP_001025328).

\section{COMT mRNA quantitation}

COMT mRNA expression levels in the brain, lung, liver, kidney and small intestine from 6 male marmosets (2-6 years of age) were measured by real-time RT-PCR. Total RNA from each pooled tissue and the first-strand cDNA were prepared as described above. PCR was performed with Power SYBR Green PCR Master Mix (Applied Biosystems) on an ABI PRISM 7300 sequence detection system (Applied Biosystems). PCR conditions were 10 min at $95^{\circ} \mathrm{C}$, followed by 40 cycles of $95^{\circ} \mathrm{C}$ for $15 \mathrm{sec}$ and $60^{\circ} \mathrm{C}$ for $1 \mathrm{~min}$. Primers were designed to measure expression level of membrane-bound and soluble COMT mRNAs and used at a final concentration of $400 \mathrm{nM}$, including cjCOMT (5qrt1) 5'-CCTTATTGTCTGGAGCGAGT-3' and cjCOMT (3qrt1) 5'-CAGTAGGTGTCAATGGCCTC-3'. COMT mRNA expression level in each tissue was determined by three independent experiments using standard curves generated by serial dilutions of marmoset COMT cDNA and normalized to 18S ribosomal RNA level measured using Eukaryotic 18S rRNA Endogenous Control (Applied Biosystems) according to the manufacturer's instructions.

\section{Immunoblot analysis}

COMT and $\beta$-actin were detected in marmoset tissue homogenates by immunoblotting using anti-human COMT antibodies and anti-avian $\beta$-actin antibodies, respectively. Marmoset tissue homogenates $(50 \mu \mathrm{g})$ were fractionated by electrophoresis on $12 \%$ sodium dodecyl sulphate-polyacrylamide gel and transferred onto polyvinylidene difluoride membranes (Merck Millipore, Billerica, MA, U.S.A.). Membranes were incubated for $30 \mathrm{~min}$ in blocking solution, $0.5 \%$ nonfat dry milk in TBS (50 mM Tris, 


\section{Marmoset chromosome 1}

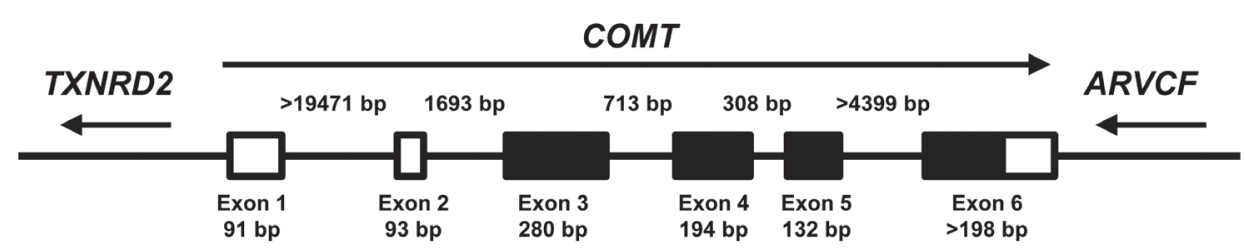

Human chromosome 22

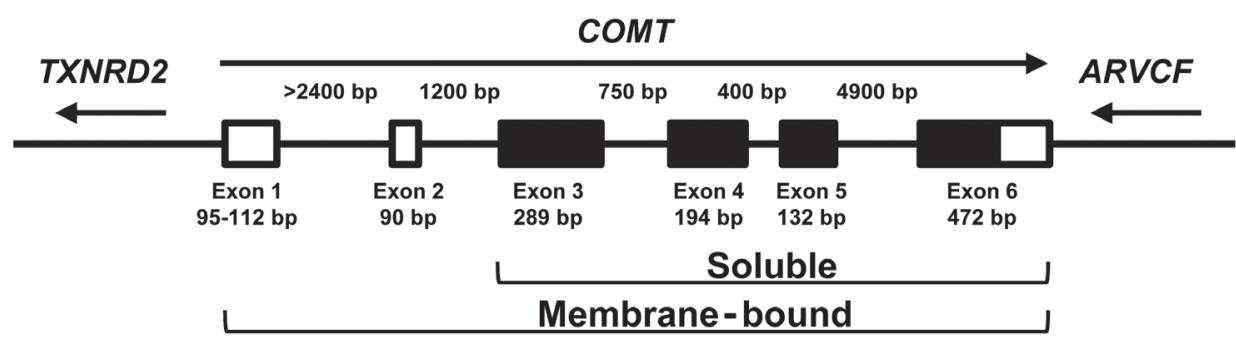

Fig. 1. Gene structures of marmoset COMT. The exon and intron sequences of marmoset COMT were determined by the BLAT analysis of the genome data using human COMT mRNA sequence (NM_000754). Closed and open squares indicate the coding and untranslated region, respectively. In marmosets and humans, COMT was located adjacent to TXNRD2 and $A R V C F$ in the corresponding genome region. The gene structure of human COMT was cited from a previous report [14].

$138 \mathrm{mM} \mathrm{NaCl}$ and $2.7 \mathrm{mM} \mathrm{KCl})$ containing $0.05 \%$ Tween $20(\mathrm{v} / \mathrm{v})$, and probed at room temperature with primary antibodies $(1: 1,000)$ for $1 \mathrm{hr}$ and subsequently with secondary antibodies $(1: 5,000)$ for $20 \mathrm{~min}$. Immunoreactive proteins were visualized using an ECL Prime Western Blotting Detection System (GE Healthcare, Buckinghamshire, U.K.).

\section{RESULTS}

By searching the marmoset genome sequences using the coding sequence of human COMT, a marmoset COMT gene was found on marmoset chromosome 1 (chr1: 184388215-184395727) (Fig. 1). To isolate marmoset COMT cDNA, RT-PCR was carried out using total RNA from marmoset liver. We successfully isolated a cDNA containing coding region (804 bp) of marmoset COMT, and the sequence has been deposited to GenBank (accession number KY088283). The deduced amino acid sequences (268 residues) contained a transmembrane region and critical amino acid residues for $\mathrm{Mg}^{2+}$-binding (Asp216 and Asn217) and adeninebinding (Leu138, His189 and Trp190) and catalytic Lys191 (Fig. 2), important residues for human COMT function [1, 2], and showed high degrees of identity $(\geq 87 \%)$ to those of humans and cynomolgus monkeys, compared with other experimental animals used in drug development (Table 1). Phylogenetic analysis indicated that marmoset COMT was grouped into the same clade as human and cynomolgus monkey COMT (Fig. 3), suggesting the evolutionary closeness of COMT in primates.

To analyze tissue distribution of COMT mRNA and protein, quantitative RT-PCR and immunoblotting were performed, respectively. COMT mRNA was expressed in all five marmoset tissues examined and was more abundant in the liver and kidney (Fig. 4). By immunoblotting, membrane-bound COMT protein of $\sim 29 \mathrm{kDa}$ was also detected in the liver and kidney (Fig. 5A). In contrast, soluble COMT protein of $\sim 24 \mathrm{kDa}$ was only detected in the liver (Fig. 5A). Membrane-bound and soluble COMT proteins were consistently expressed in the liver of individual marmosets (Fig. 5B).

\section{DISCUSSION}

MPTP-treated marmosets have been used as animal models to develop inhibitory drugs on COMT, which would extend the therapeutic time window of levodopa for Parkinson's disease. However, the molecular characteristics of marmoset COMT have not been fully investigated. In this study, we identified novel marmoset COMT cDNA from livers. Marmoset COMT showed highly similar amino acid sequences (90\%) to human COMT, compared with those of rodents (77-78\%) widely used in preclinical studies. (Table 1). Phylogenetic analysis also showed that marmoset COMT has a closer evolutionary relationship to human COMT than those of rodents (Fig. 3).

It has been reported previously that COMT is located in many tissues of various species. Generally, COMT protein was most abundant in the liver of humans, rats and mice $[9,10,14]$. In rats, soluble COMT protein was most abundant in the liver, followed by the kidney, thymus, spleen and lung, but in addition to these tissues, membrane-bound COMT was also expressed in the brain [10]. In humans, soluble COMT protein was most abundant in the liver, followed by the adrenal, placenta, kidney and duodenum, 


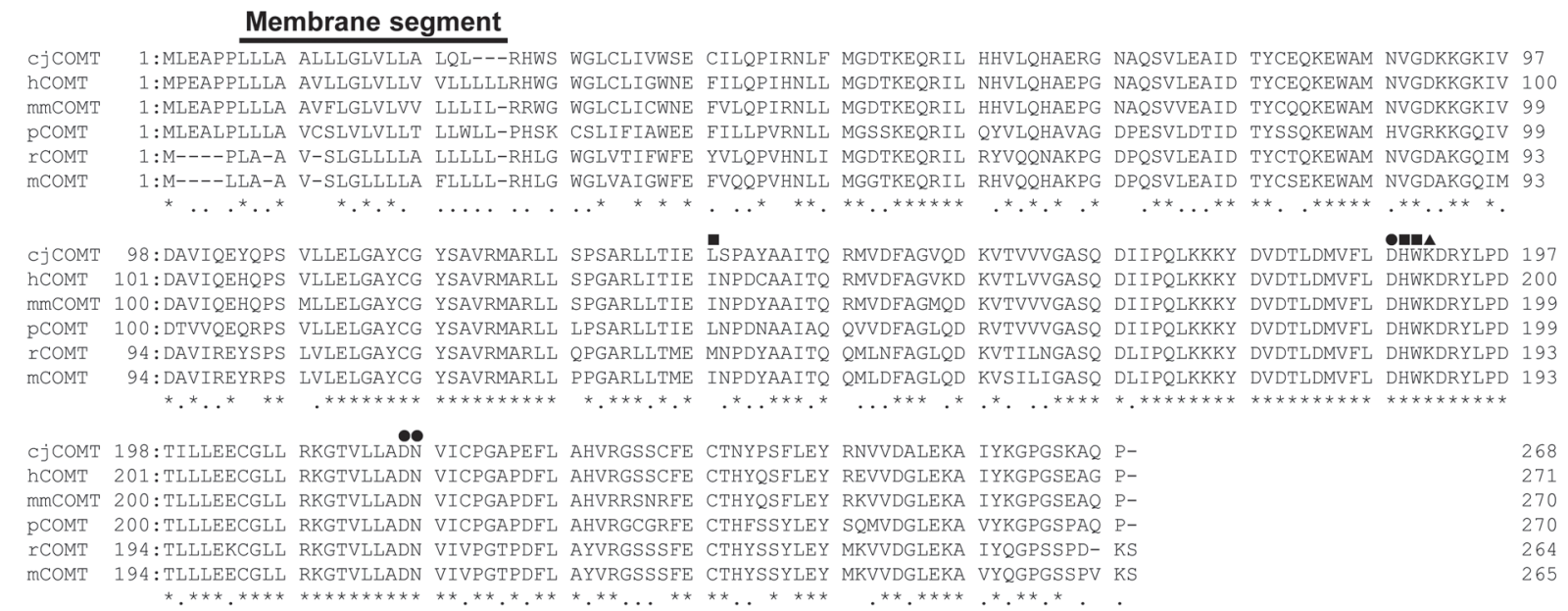

Fig. 2. Alignment of the amino acid sequences of marmoset COMT. Amino acid sequences of marmosets (cj), humans (h), rhesus monkeys $(\mathrm{mm})$, pigs (p), rats (r) and mice (m) were aligned, as described in Materials and Methods. Solid line indicates the $N$-terminal transmembrane sequences. Circles and squares indicate the residues in the adenine and $\mathrm{Mg}^{2+}$-binding sites, respectively. A triangle indicates the catalytic lysine residue. Asterisks and dots indicate amino acid residues completely and relatively conserved among the species examined, respectively.

Table 1. Sequence identity of cDNA and amino acids of marmoset COMT compared with other species

\begin{tabular}{lcc}
\hline \multirow{2}{*}{ Species } & \multicolumn{2}{c}{ Identity (\%) } \\
\cline { 2 - 3 } & cDNA & Amino acids \\
\hline Human & 90 & 90 \\
Rhesus monkey & 90 & 87 \\
Pig & 80 & 77 \\
Rat & 80 & 78 \\
Mouse & 79 & 77 \\
\hline
\end{tabular}

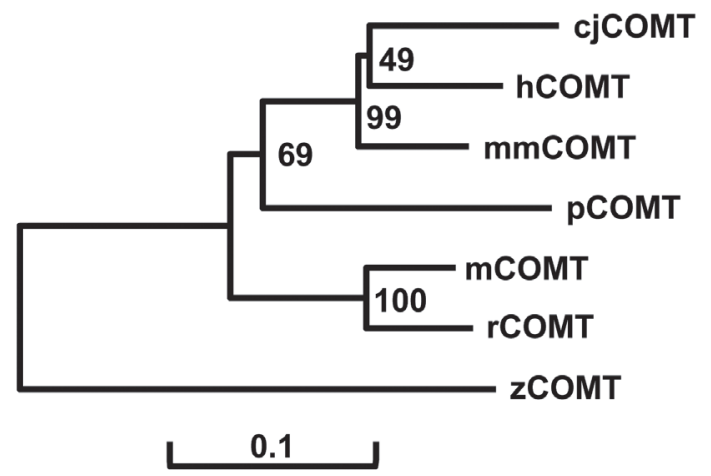

Fig. 3. Phylogenetic comparison of amino acid sequences of COMT from various species. The phylogenetic tree was constructed by the neighbor-joining method using membrane-bound COMT amino acid sequences from marmosets (cj), humans (h), rhesus monkeys (mm), pigs (p), rats (r) and mice (m). COMT amino acid sequences from zebrafish (z) was used as an outgroup. The number at each node indicates the bootstrap value supporting the node. The scale bar marks 0.1 amino acid substitution per site.

but in addition to these tissues, membrane-bound COMT protein was also expressed in the brain [14]. Similarly, in marmosets, COMT mRNA was expressed in all five tissues tested, but was relatively more abundant in liver and kidney (Fig. 4). We were not able to detect distinguishably MB- and S-COMT mRNAs even when COMT mRNA quantitation was carried out with primers designed based on N- and C-terminal sequences of COMT cDNA cloned (Uehara, unpublished data). There is a possibility that MB- and S-COMT are translated from common or similar transcripts. In humans, it is not well known about the transcriptional and translational regulation of MB- and S-COMT. It is of great interest to investigate alternative splicing, alternative promoter usage and/or post-translational modification for generating protein diversity and regulating tissue-type specific expression of marmoset COMT. Marmoset membrane-bound and soluble COMT proteins were only detected in the liver/kidney and liver, respectively (Fig. 5A). MB- and S-COMT proteins were not detected in the marmoset brain unlike a previous report (Zeng et al., 2010), possibly due to the differences in the sensitivity of the COMT antibodies used. Moreover, a previous report indicated that membrane-bound and soluble COMT proteins were expressed in the livers and brains from marmosets [17]. A similar tissue distribution, together with high sequence identity and close phylogenetic relationship, suggests molecular similarity of COMT between marmosets and humans. Thus, the marmoset would be a non-human primate model suitable for drug development.

It has been understood that the two isoforms of COMT have, at least partially, distinct roles [7]. Membrane-bound COMT is suggested to be involved in the termination of dopaminergic and noradrenergic synaptic neurotransmission [12]. Membrane-bound 


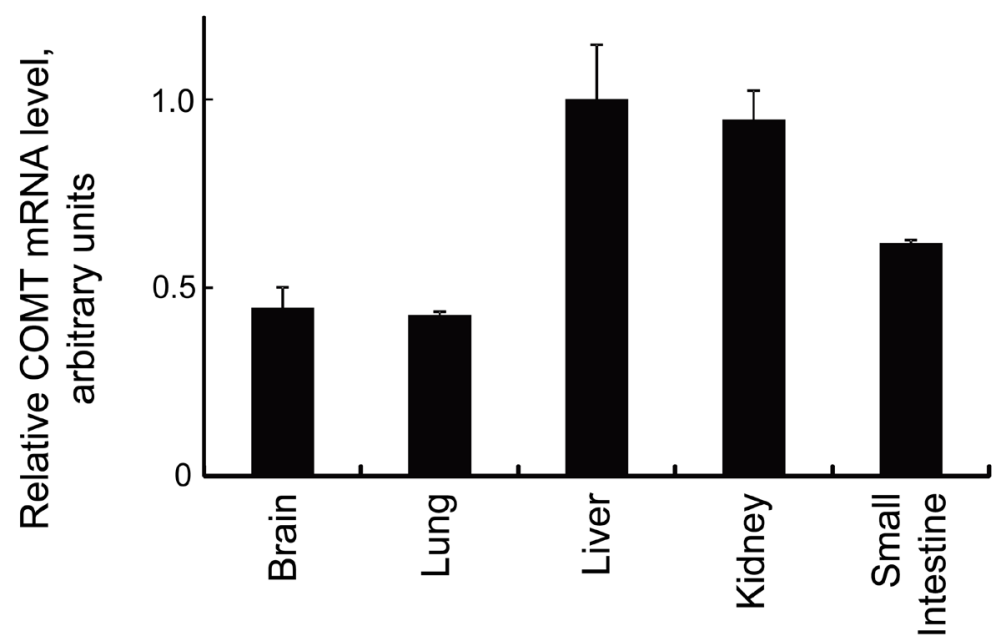

Fig. 4. Distribution of COMT mRNA in marmoset tissues. Expression levels of marmoset COMT mRNA were normalized to $18 \mathrm{~S}$ ribosomal RNA levels and represent the averages $\pm \mathrm{SD}$ from three independent experiments. The expression level in each tissue is indicated as relative values to that in the liver.

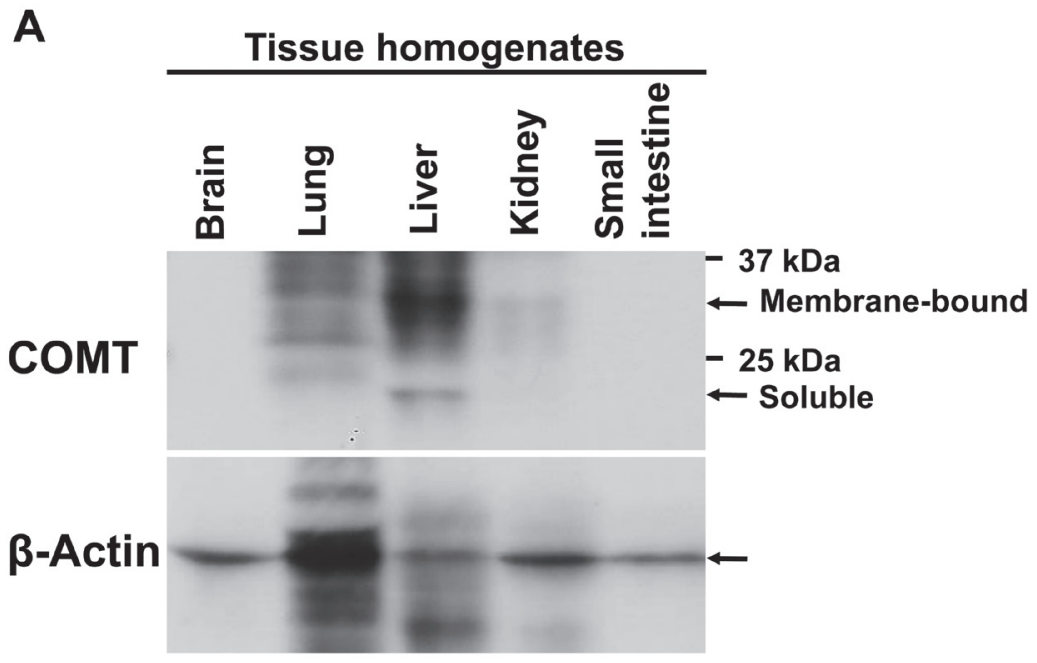

B

Individual liver homogenates

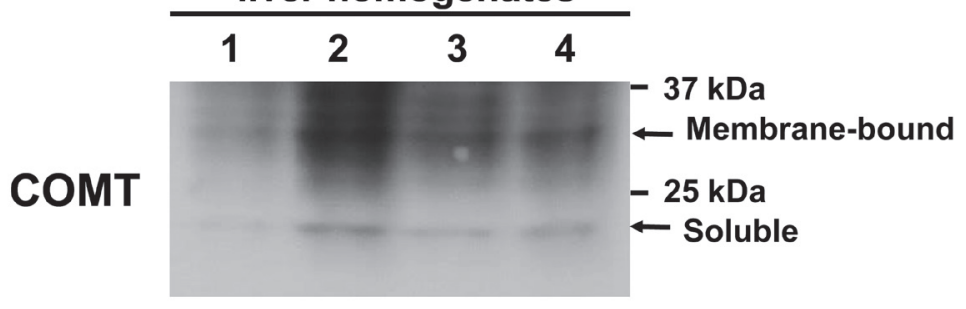

Fig. 5. Immunoreactive detection of COMT in tissue homogenates from marmosets. Membrane-bound and soluble COMTs were detected by immunoblotting using anti-human COMT antibodies in pooled tissue homogenates (A) and individual liver homogenates (B). $\beta$-Actin was used as an internal control.

COMT has a high affinity for catecholamine substrates and dopamine, but a lower activity than soluble COMT [6]. In contrast, soluble COMT has a high capacity and is suggested to mainly contribute to the inactivation of xenobiotic catechols. In COMT amino acid sequences, critical functional residues including adenine-binding sites, $\mathrm{Mg}^{2+}$-binding sites and a catalytic lysine residue were relatively conserved among various species (Fig. 2). However, an active site of marmoset COMT may differ from human and rhesus monkey COMT forms, because the 148th amino acid residue was Leu for marmosets and Ile for humans and rhesus 
monkeys. This sequence difference might cause species differences in the substrate selectivity of COMT forms between marmosets and rhesus monkeys/humans. Further investigation on catalytic function using recombinant proteins and tissue microsomes/cytosols would help the understanding of COMT-dependent metabolism in marmosets.

In conclusion, the novel marmoset COMT was identified by the analysis of the marmoset genome data. Marmoset COMT cDNA was isolated from liver by RT-PCR. The deduced amino acid sequences were highly identical (87-90\%) to those of rhesus monkey and human COMTs and showed the evolutionary closeness to rhesus monkey and human COMTs. Marmoset COMT mRNA was expressed in various tissues. Membrane-bound and soluble COMT proteins were abundantly expressed in livers. The similarities in the primary sequence features and tissue expression patterns of marmoset COMT to human COMT suggest similar molecular characteristics of COMT between these two species.

DECLARATION OF INTEREST. This work resulted from "Construction of System for Spread of Primate Model Animals" under the Strategic Research Program for Brain Sciences of the Japan Agency for Medical Research and Development. S.U. was also supported partly by the Japan Society for the Promotion of Science Grant-in-Aid for Young Scientists B [15K18934]. The authors are responsible for the content and writing of the article and report no declarations of interest.

ACKNOWLEDGMENTS. The authors thank Drs. Norie Murayama and Makiko Shimizu for their technical help, and Mr. Lance Bell for advice on English writing.

\section{REFERENCES}

1. Chen, J., Song, J., Yuan, P., Tian, Q., Ji, Y., Ren-Patterson, R., Liu, G., Sei, Y. and Weinberger, D. R. 2011. Orientation and cellular distribution of membrane-bound catechol-O-methyltransferase in cortical neurons: implications for drug development. J. Biol. Chem. 286: 34752-34760. [Medline] [CrossRef]

2. Ehler, A., Benz, J., Schlatter, D. and Rudolph, M. G. 2014. Mapping the conformational space accessible to catechol-O-methyltransferase. Acta Crystallogr. D Biol. Crystallogr. 70: 2163-2174. [Medline] [CrossRef]

3. Karhunen, T., Tilgmann, C., Ulmanen, I., Julkunen, I. and Panula, P. 1994. Distribution of catechol-O-methyltransferase enzyme in rat tissues. J. Histochem. Cytochem. 42: 1079-1090. [Medline] [CrossRef]

4. Kishi, N., Sato, K., Sasaki, E. and Okano, H. 2014. Common marmoset as a new model animal for neuroscience research and genome editing technology. Dev. Growth Differ. 56: 53-62. [Medline] [CrossRef]

5. Lautala, P., Ulmanen, I. and Taskinen, J. 2001. Molecular mechanisms controlling the rate and specificity of catechol $O-$ methylation by human soluble catechol O-methyltransferase. Mol. Pharmacol. 59: 393-402. [Medline]

6. Lotta, T., Vidgren, J., Tilgmann, C., Ulmanen, I., Melén, K., Julkunen, I. and Taskinen, J. 1995. Kinetics of human soluble and membrane-bound catechol $O$-methyltransferase: a revised mechanism and description of the thermolabile variant of the enzyme. Biochemistry 34: $4202-4210$. [Medline] [CrossRef]

7. Männistö, P. T. and Kaakkola, S. 1999. Catechol-O-methyltransferase (COMT): biochemistry, molecular biology, pharmacology, and clinical efficacy of the new selective COMT inhibitors. Pharmacol. Rev. 51: 593-628. [Medline]

8. Marvanová, M., Ménager, J., Bezard, E., Bontrop, R. E., Pradier, L. and Wong, G. 2003. Microarray analysis of nonhuman primates: validation of experimental models in neurological disorders. FASEB J. 17: 929-931. [Medline]

9. Myöhänen, T. T., Schendzielorz, N. and Männistö, P. T. 2010. Distribution of catechol-O-methyltransferase (COMT) proteins and enzymatic activities in wild-type and soluble COMT deficient mice. J. Neurochem. 113: 1632-1643. [Medline]

10. Øverbye, A. and Seglen, P. O. 2009. Phosphorylated and non-phosphorylated forms of catechol $O$-methyltransferase in rat liver, brain and other tissues. Biochem. J. 417: 535-545. [Medline] [CrossRef]

11. Philippens, I. H., 't Hart, B. A. and Torres, G. 2010. The MPTP marmoset model of parkinsonism: a multi-purpose non-human primate model for neurodegenerative diseases. Drug Discov. Today 15: 985-990. [Medline] [CrossRef]

12. Roth, J. A. 1992. Membrane-bound catechol-O-methyltransferase: a reevaluation of its role in the O-methylation of the catecholamine neurotransmitters. Rev. Physiol. Biochem. Pharmacol. 120: 1-29. [Medline]

13. Smith, L. A., Jackson, M. J., Al-Barghouthy, G., Rose, S., Kuoppamaki, M., Olanow, W. and Jenner, P. 2005. Multiple small doses of levodopa plus entacapone produce continuous dopaminergic stimulation and reduce dyskinesia induction in MPTP-treated drug-naive primates. Mov. Disord. 20: 306-314. [Medline] [CrossRef]

14. Tenhunen, J., Salminen, M., Lundström, K., Kiviluoto, T., Savolainen, R. and Ulmanen, I. 1994. Genomic organization of the human catechol O-methyltransferase gene and its expression from two distinct promoters. Eur. J. Biochem. 223: 1049-1059. [Medline] [CrossRef]

15. Uno, Y., Uehara, S. and Yamazaki, H. 2016. Utility of non-human primates in drug development: Comparison of non-human primate and human drug-metabolizing cytochrome P450 enzymes. Biochem. Pharmacol. S0006-2952(16)30134-4 (In press). [Medline]

16. Yun, J. W., Ahn, J. B. and Kang, B. C. 2015. Modeling Parkinson's disease in the common marmoset (Callithrix jacchus): overview of models, methods, and animal care. Lab. Anim. Res. 31: 155-165. [Medline] [CrossRef]

17. Zeng, B. Y., Balfour, R. H., Jackson, M. J., Rose, S. and Jenner, P. 2010. Expression of catechol- $O$-methyltransferase in the brain and periphery of normal and MPTP-treated common marmosets. J. Neural Transm. (Vienna) 117: 45-51. [Medline] [CrossRef]

18. Zubair, M., Jackson, M. J., Tayarani-Binazir, K., Stockwell, K. A., Smith, L. A., Rose, S., Olanow, W. and Jenner, P. 2007. The administration of entacapone prevents L-dopa-induced dyskinesia when added to dopamine agonist therapy in MPTP-treated primates. Exp. Neurol. 208: 177-184. [Medline] [CrossRef] 